\title{
Adsorbate motions induced by inelastic-tunneling current: Theoretical scenarios of two-electron processes
}

\author{
H. Ueba ${ }^{\text {a) }}$ and T. Mii \\ Department of Electronics, Toyama University, Toyama, 930-8555, Japan
}

\author{
N. Lorente \\ Laboratoire Collisions, Agrégats, Réactivité, Unité Mixte de Recherche (UMR) 5589, Institut de Recherche \\ sur les Systèmes Atomiques et Moléculaires Complexes (IRSAMC), Université Paul Sabatier, \\ 118 Route de Narbonne, F-31062 Toulouse Cedex 4, France
}

B. N. J. Persson

Institut fur Festkorperforschung, Forschungszentrum Jülich, D-52425 Jülich, Germany

(Received 1 December 2004; accepted 30 June 2005; published online 31 August 2005)

\begin{abstract}
We discuss how the excitation of high-frequency modes in adsorbed molecules may result in motion (e.g., rotation, translation, or dissociation) of the molecules. Our study is based on rate equations and considers one- and two-vibrational excitation processes, corresponding to linear and quadratic dependences of the reaction rate on the tunneling current in the case the scanning tunneling microscopy is used to excite the vibrations (inelastic tunneling). From the results reported in this paper it should be possible to obtain intramolecular transition rates directly from the experimental data, and gain some understanding on how these important quantities depend on the modes involved and on the substrate. () 2005 American Institute of Physics. [DOI: 10.1063/1.2007631]
\end{abstract}

\section{INTRODUCTION}

Using the scanning tunneling microscope (STM), inelastic tunneling may be used to selectively excite the vibrational modes of single molecules. ${ }^{1}$ It has been shown that single- or multiple-vibrational excitation of atoms or molecules adsorbed on surfaces may result in adsorbate motions and reactions. ${ }^{2-7}$ Figure 1 shows schematically the vibrational ladder climbing by a direct excitation of a reaction coordinate $(\mathrm{RC})$ mode by tunneling electrons (green arrow). Incoherent vibrational ladder climbing ${ }^{8-10}$ explains the Xe-atom transfer between a STM tip and the substrate. ${ }^{11}$ This theory has also been applied to explain local bond breaking for hydrogen desorption from the $\mathrm{H}-\mathrm{Si}(100)$ system. ${ }^{12}$ Coherent vibrational ladder climbing, ${ }^{13}$ resulting from direct excitation of the $\mathrm{O}-\mathrm{O}$ stretch mode or the $\mathrm{C}-\mathrm{H}$ stretch mode, has been suggested as the mechanism for the dissociation of an oxygen molecule on $\mathrm{Pt}(111)$ (Ref. 14) and the transformation from trans-butene to 1,3-butadiene molecule on $\operatorname{Pd}(110){ }^{15}$ In both mechanisms the reaction rate is characterized by the power-law dependence on tunneling current, with the power exponent representing the number of inelastic-tunneling electrons required to overcome the potential barrier $E_{B}$.

When the energy stored in a high-frequency (HF) mode excited by tunneling electrons (black arrow in Fig. 1) is higher than the potential barrier $E_{B}$ along RC of a particular adsorbate motion, energy transfer via anharmonic coupling from the HF mode to the RC mode (white arrow) may induce adsorbate motion. This indirect single-electron process was applied to explain the lateral hopping of $\mathrm{CO}$ on $\operatorname{Pd}(110)$

\footnotetext{
${ }^{a)}$ Author to whom correspondence should be addressed. Electronic mail: ueba@eng.toyama-u.ac.jp
}

(Ref. 16) and the rotation of acetylene on $\mathrm{Cu}(100),{ }^{17}$ where the excitation of the $\mathrm{C}-\mathrm{O}(\hbar \Omega=240 \mathrm{meV})$ and the $\mathrm{C}-\mathrm{H}$ stretch mode $(\hbar \Omega=358 \mathrm{meV})$ were found to trigger these motions. The theory ${ }^{18}$ has clarified how the energy stored in the HF modes is transferred to the RC modes to activate their motions $\left(E_{B}=150 \mathrm{meV}\right.$ for $\mathrm{C}-\mathrm{O}$ hopping and $170 \mathrm{meV}$ for $\mathrm{C}_{2} \mathrm{H}_{2}$ rotation).

Stipe et al. have made a systematic experimental study of the inelastic-tunneling-induced rotation of an oxygen molecule on $\mathrm{Pt}(111) .{ }^{19}$ The rotation rate as a function of tunneling current for several fixed bias voltage $(0.1-0.3 \mathrm{~V})$ was found to follow a power law $R \propto I^{n}$. At the bias voltage of $0.1 \mathrm{~V}$, two electrons were required to induce rotational motion. Knowing that $\hbar \Omega=87 \mathrm{meV}$ for the $\mathrm{O}-\mathrm{O}$ stretch mode,

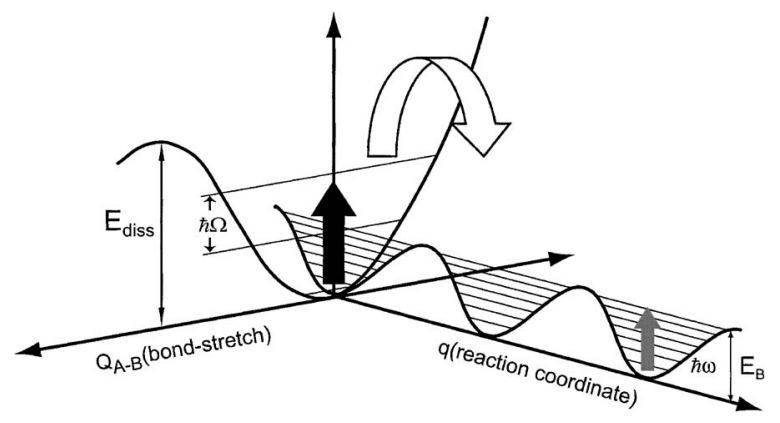

FIG. 1. Schematics of a simple two-dimensional potential along the intramolecular stretch $Q_{A-B}$ and along the reaction coordinate $q$. It is implicitly assumed that the barrier for the $A-B$ bond-breaking (dissociation) $E_{\text {diss }}$ is higher than the barrier $E_{B}$ associated with the RC mode. There are two ways to overcome $E_{B}$ : (a) Coherent or incoherent vibrational ladder climbing (green arrow) involving direct excitation of the RC mode by tunneling electrons. (b) If $\hbar \Omega>E_{B}$ anharmonic coupling between the high-frequency $A-B$ stretch mode excited by tunneling electrons (black arrow) and the lowfrequency RC mode allows the excitation of the RC mode above $E_{B}$. 
$\hbar \omega=37 \mathrm{meV}$ for the frustrated rotation mode and $E_{B}=150-175 \mathrm{meV}$ for the rotation barrier, rotation may be induced by the excitation of the $\mathrm{O}-\mathrm{O}$ stretch mode by a two-electron process. However, this requires an anharmonic coupling between the two modes. This also implies that the energy transfer via anharmonic coupling dominates over the direct excitation of the frustrated rotation mode by many tunneling electrons. Furthermore, the molecule finally dissociates at the same voltages $(0.2-0.4 \mathrm{~V})$ that induced the rotation. Ho suggested that the $\mathrm{O}_{2}$ molecule is rotating when it is being excited by tunneling electrons to dissociate. ${ }^{20}$ Since the rotation barrier is lower than the dissociation barrier $(380 \mathrm{meV})$, it is easier to excite the rotation. Ho speculated that the rotation may be coupled to the dissociation, but there is no direct experimental evidence to support his claim. Indeed, Teillet-Billy et $a l^{21}$ proposed a theory assuming that one can induce $\mathrm{O}_{2}$ rotation directly by the tunneling electrons. It is still an open question how one can control the rotation or dissociation with a suitable combination of tunneling current and bias voltage. A search of the threshold bias voltage above which rotation or dissociation can be induced at fixed tunneling current is expected to provide more insights into the elementary processes responsible for different motions of $\mathrm{O}_{2}$ on $\mathrm{Pt}(111)$.

Recently Pascual et al. ${ }^{22}$ reported that they could selectively excite vibrational modes in an ammonia molecule $\left(\mathrm{NH}_{3}\right)$ on $\mathrm{Cu}(100)$ as a way to break the chemical bond between $\mathrm{NH}_{3}$ and the copper surface, or alternatively to induce the molecule to move laterally on the surface. The reaction outcome was controlled by selecting the bias voltage so that the energy of the tunneling electron was enough to excite the preferred vibrational mode and the tunneling current was modified to select the excitation rate. The two modes investigated are the N-H stretch mode, and the umbrellalike bending mode. Although most of the vibrational excited states quickly relax by transferring their energy to the substrate, a small fraction of the vibrational excited molecules convert their vibrational energy into hopping or desorption from the surface. They found that translation, with the activation barrier of $E_{B}=300 \mathrm{meV}$, is induced by a single excitation of the $\mathrm{N}-\mathrm{H}$ stretch mode $(408 \mathrm{meV})$, while the double excitation of the $\mathrm{N}-\mathrm{H}$ mode (two-electron process) is required to desorb the molecule $\left(E_{B}=600 \mathrm{meV}\right)$. Thus the indirect process involving the vibrational excitation of the HF modes by tunneling electrons dominates over the direct multiple excitations of the relevant low-frequency RC modes. Selective control of single-molecule motions by tunning tunneling currents and electron energies has also been reported by Kim et al. ${ }^{23}$ for rotation and hopping of $\mathrm{C}_{2} \mathrm{H}_{2}$ on $\mathrm{Pd}(111)$. These sophisticated experiments not only reveal how competing reaction pathways can be selectively activated within a single molecule, but also demonstrates a new approach for identifying the reaction pathways in complex systems.

The purpose of this work is to discuss single- and twoelectron processes for adsorbate motions induced by the vibrational excitation of the HF mode and/or the RC mode. The present discussion does not focus on any particular experimental situations. We present coupled rate equations de- scribing the evolution of the vibrational population of the HF mode and of the RC mode. With a reaction we mean a process by which the RC mode is excited above the barrier. The equations are solved to obtain the reaction rates by singleand two-electron processes. This allows us to study how the reaction rates depend on the inelastic-tunneling fraction, the anharmonic intermode couplings, and on the vibrational dampings.

The reaction rate $R_{\mathrm{RC}}$ can be written as the product of the excitation rate $\Gamma_{\mathrm{RC}(m \rightarrow n)}$ times the probability $N_{\mathrm{HF}(\nu), \mathrm{RC}(m)} \equiv N_{\nu, m}$ that the HF mode is in state $\nu(\nu=0,1,2)$, and the RC mode is in state $m(m=0,1,2, \ldots)$ :

$$
R_{\mathrm{RC}}=\sum_{m, n} \Gamma_{\mathrm{RC}(m \rightarrow n)} N_{\nu, m} .
$$

Here $n$ stands for the vibrational continuum of the RC mode above the barrier $E_{B}$. When the vibrational energy $\hbar \Omega$ of the $\mathrm{HF}$ mode is larger than $E_{B}$, a reaction can occur in a singleelectron process, where the HF mode decays by transferring energy to the RC mode via anharmonic interaction, resulting in the excitation of the RC mode from the ground state to a state above the barrier. In this case $\Gamma_{\mathrm{RC}(m \rightarrow n)}$ is the rate of energy transfer from the HF mode to the RC mode and $\nu$ $=1$ and $m=0$ in Eq. (1).

If $2 \hbar \Omega>E_{B}>\hbar \Omega$ the RC mode can be excited above $E_{B}$ either by the decay of the $\nu=1$ state excited twice, or by energy transfer from the $\nu=2$ state of the HF mode (populated by ladder climbing from the $\nu=1$ state). These indirect excitation processes correspond to $N_{\mathrm{HF}(v), \mathrm{RC}(m)}=N_{1, m}$ and $N_{2,0}$, respectively. Furthermore, if $\hbar \Omega+m \hbar \omega>E_{B}$ (where $m$ denotes the intermediate excited states of the RC mode) a cooperative process leading to a reaction is possible where both the HF and the RC modes are excited by tunneling electrons. In addition to these processes mediated by the anharmonic coupling between the HF and the RC modes, a direct excitation of the RC mode above $E_{B}$ without involving the HF mode is also possible. Dependent on the relative time scale between the rate of vibrational damping and the inelastic tunneling, the theory of incoherent multiple single-step vibrational ladder climbing, ${ }^{8-10}$ and coherent multiplevibrational excitation ${ }^{13}$ have been successfully applied to explain dynamical processes of adsorbed atoms and molecules induced by inelastic-tunneling electrons.

In the following the rate equations for the level populations $N_{\mathrm{HF}(\nu), \mathrm{RC}(m)}$ are solved to obtain the reaction rate by single- and two-electron processes. In particular, we explore all the possible two-electron processes, which are characterized by the fraction of tunneling electrons that lead to vibrational excitation (inelastic-tunneling fraction), the strength of the anharmonic mode coupling, and the vibrational damping rates of the $\mathrm{HF}$ and the RC modes. This allows us to examine what type of the two-electron process dominates depending on these key parameters.

\section{SINGLE-ELECTRON PROCESS}

Experiments have shown that the excitation of the $\mathrm{C}-\mathrm{O}$ stretch vibration for $\mathrm{CO}$ on $\mathrm{Pd}(110)$ and the $\mathrm{N}-\mathrm{H}$ vibration for $\mathrm{NH}_{3}$ on $\mathrm{Cu}(100)$ can lead to transnational motion of the 


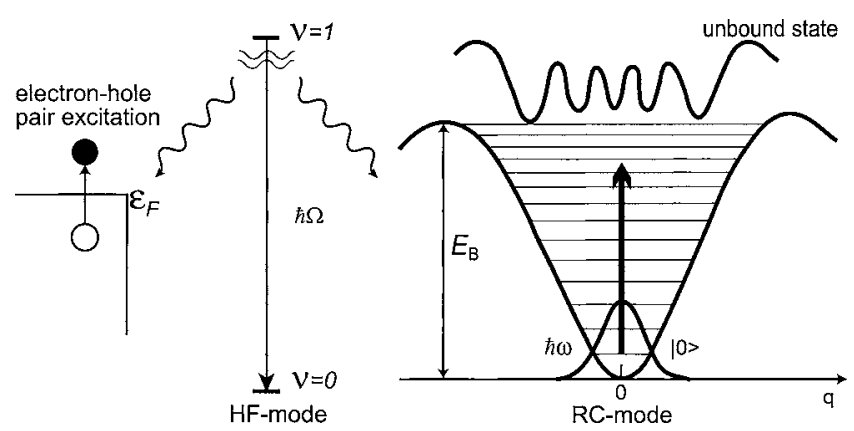

FIG. 2. Schematic illustration of a single-electron process. The decay of the high-frequency (HF) mode with the energy $\hbar \Omega \geqslant E_{B}$ excites the lowfrequency reaction coordinate mode from the ground state localized at the bottom of the RC-potential well to the unbound state just above the top of the barrier, in competing with EHP excitations.

molecules. ${ }^{17,22}$ Similarly, excitation of the $\mathrm{C}-\mathrm{H}$ mode for $\mathrm{C}_{2} \mathrm{H}_{2}$ on $\mathrm{Cu}(100)$ can induce the rotation of the molecule. ${ }^{18}$ A common feature of these systems is the appearance of a threshold bias voltage (corresponding to the $\mathrm{C}-\mathrm{O}, \mathrm{N}-\mathrm{H}$, and $\mathrm{C}-\mathrm{H}$ vibrational excitations) above which transnational or rotational motion of the molecules is induced.

Figure 2 depicts a single-electron process where anharmonic mode coupling between the HF mode (excited by tunneling electron) and the RC mode results in the excitation of the RC mode to a level above $E_{B}$. We will denote this process as

$$
[\nu: 0 \rightarrow 1](\nu: 1 \rightarrow 0 \mid \mathrm{RC}: 0 \rightarrow n) .
$$

Here $[\nu: 0 \rightarrow 1]$ stands for a process where a tunneling electron excites the HF mode $\nu$ from the ground state $\nu=0$ to the first excited state $\nu=1$. Also, $(\nu: 1 \rightarrow 0 \mid \mathrm{RC}: 0 \rightarrow n)$ denote a process where the HF mode decays from its first vibrational excited state $\nu=1$ to the ground state $\nu=0$ while the RC mode is excited from the ground state to a level $n$ above the barrier $E_{B}$. This transfer of energy from the HF mode to the the RC mode $n$ is possible because of the anharmonic coupling between the two modes. In general, this process may involve depositing some energy $\epsilon$ in the continuum of electron-hole pair (EHP) excitations (for metals) or bulk phonon excitations. Thus, in general $\hbar \Omega=\hbar \omega_{n}+\epsilon$.

The reaction rate $R_{\mathrm{RC}}$ induced by the single-electron process schematically depicted in Fig. 2 is

$$
R_{\mathrm{RC}}=\sum_{n>n_{\mathrm{th}}} \frac{1}{\tau_{\nu(1 \rightarrow 0) \mathrm{RC}(0 \rightarrow n)}} N_{1,0},
$$

where

$$
\frac{1}{\tau_{\nu(1 \rightarrow 0) \mathrm{RC}(0 \rightarrow n)}}
$$

is the transition rate of deexciting the $\nu$ mode from its first excited state to the ground state while exciting the RC mode from its ground state to the higher-lying states $n$. The $n$ sum is over all the levels $n>n_{\text {th }}$ above the top of the barrier $E_{B}$ that satisfy the energy conservation $\hbar \Omega=\hbar \omega_{n}+\epsilon$. It is assumed that any frictional coupling to the surroundings does not quench the excited state above $E_{B}$ before the molecule has been able to pass the top of the potential barrier. Here we have also neglected thermal effects. The calculation of $\tau_{\nu, \mathrm{RC}}$ requires, in general, a detailed knowledge of the potentialenergy surface of the system.

We also obtain

$$
\frac{d N_{1,0}}{d t}=p_{\nu} N_{0,0}-\frac{1}{\tau_{\nu}} N_{1,0}-\sum_{n} \frac{1}{\tau_{\nu(1 \rightarrow 0) \mathrm{RC}(0 \rightarrow n)}} N_{1,0},
$$

where $p_{\nu}$ is the transition rate to excite the $\nu=1$ state by the inelastic tunneling, and is written as $p_{\nu}=\eta_{\nu} I$ in terms of the inelastic-tunneling fraction ${ }^{24} \eta_{\nu}$ and the electron-tunneling rate or current $I$. The vibrational damping $\tau_{\nu}$ is due to lowenergy excitations (EHP excitations ${ }^{25,26}$ and bulk phonons). Equation (3) and the approximate condition $N_{0,0}=1$ used below are based on the assumption that $p_{\nu} \tau_{\nu} \ll 1$, otherwise one has to include the population of higher vibrational states and electron-tunneling-induced deexcitation.

The reaction rate can be calculated by assuming a stationary state so that $d N_{1,0} / d t=0$, which gives

$$
N_{1,0}=\widetilde{\tau}_{\nu} p_{\nu} N_{0,0},
$$

where the total relaxation rate of the first excited state of the $\nu$ mode is given by

$$
\frac{1}{\widetilde{\tau}_{\nu}}=\frac{1}{\tau_{\nu}}+\sum_{n} \frac{1}{\tau_{\nu(1 \rightarrow 0) \mathrm{RC}(0 \rightarrow n)}} .
$$

Using Eqs. (3) and (6) and the condition $N_{0,0}=1$ leads to the reaction rate

$$
\begin{aligned}
& R_{\mathrm{RC}}=\frac{\tilde{\tau}_{\nu}}{\tau_{\nu, \mathrm{RC}}^{>}} \eta_{\nu} I, \\
& \frac{1}{\tau_{\nu, \mathrm{RC}}^{>}}=\sum_{n>n_{\mathrm{th}}} \frac{1}{\tau_{\nu(1 \rightarrow 0) \mathrm{RC}(0 \rightarrow n)}} .
\end{aligned}
$$

When the vibrational damping rate $1 / \tau_{\nu}$ is much larger than that due to the intermode coupling $1 / \tau_{\nu, \mathrm{RC}}$, Eq. (8) reduces to

$$
R_{\mathrm{RC}}=\frac{1}{\tau_{\nu, \mathrm{RC}}^{>}} \tau_{\nu} \eta_{\nu} I
$$

where $\tau_{\nu} / \tau_{\nu, \mathrm{RC}}^{>}$is the ratio of the rate of excitation of the RC mode above the potential barrier $E_{B}(\leqslant \hbar \Omega)$, and the decay rate of the $\nu$ mode into low-energy excitations in the substrate.

Equation (10) describes a process involving a single electron, and thus the number of events per electron is constant as experimentally observed for $\mathrm{CO}$ and $\mathrm{NH}_{3}$ translation on $\mathrm{Pd}(110)$ and $\mathrm{Cu}(100)$, respectively. The calculation of $1 / \tau_{\nu, \mathrm{RC}}$ depends on the model of anharmonic coupling between the $\nu$ mode excited by a tunneling electron, and the low-frequency frustrated mode (energy $\hbar \omega$ ) associated with a particular motion of a molecule. Our previous theory ${ }^{16}$ that studied how the energy stored in the HF mode is transferred to transnational or rotational energy along the RC coordinate, in competion with the fast vibrational relaxation through electron-hole pair (EHP) excitation, gives 


$$
\frac{1}{\tau_{\nu, \mathrm{RC}}^{>}} \approx \frac{1}{\tau_{\nu}}\left(\frac{\hbar \delta \omega}{E_{B}}\right)^{2} \alpha^{3 / 2} e^{-2 \alpha}
$$

where $\delta \omega$ is the anharmonic coupling between the $\nu$ mode and the low-frequency frustrated mode (translation or rotation) and $\alpha=E_{B} / \hbar \omega$. It has been shown (see Ref. ${ }^{16}$ ) that the anharmonic intermode coupling enables the RC mode to be directly excited from the ground state to an unbound continuum state above $E_{B}$. This theory agrees in the order of magnitude with the experimental result of a $\mathrm{CO}$ hopping rate on $\mathrm{Pd}(110)$. It can also explain why $\mathrm{CO}$ hopping is not observed on $\mathrm{Cu}(110)$; this follows from the small $\delta \omega$ and a large $\alpha$ [see Refs. 18 and 16 for more details of the qualitative difference between $\mathrm{CO}$ on $\mathrm{Pd}(110)$ and $\mathrm{Cu}(110)]$.

We note that for adsorbates on metals, vibrational relaxation rates are usually much larger than the intermode coupling rates $1 / \tau_{\nu, \mathrm{RC}}$. Thus, for example, for $\mathrm{CO}$ on $\mathrm{Pd}(110)$, the vibrational relaxation rate of the $\mathrm{C}-\mathrm{O}$ stretch vibration is in the order of $1 / \tau_{\nu} \simeq 10^{12} / \mathrm{s}$, while $1 / \tau_{\nu, \mathrm{RC}}^{>} \simeq 10^{3} / \mathrm{s}$, where the RC mode corresponds to lateral hopping. For adsorbates on semiconductors or insulators, however, the situation may be very different because HF modes may have long lifetimes. One extreme example is $\mathrm{CO}$ on $\mathrm{NaCl}(100)$ (Ref. 27) where the damping of the $\mathrm{C}-\mathrm{O}$ stretch mode due to the phonon excitation in the substrate is very weak, $1 / \tau_{\nu} \simeq 10^{2} / \mathrm{s}$.

We note that when the bias voltage $V$ is larger than $E_{R C} / e$, the energy of a single tunneling electron is sufficient to directly excite the RC mode above the barrier. ${ }^{14}$ In this case the single-step process ${ }^{13}$ dominates over multiple-step processes if the vibrational relaxation rate is much larger than the maximum electron-tunneling rate.

\section{TWO-ELECTRON PROCESSES}

We now consider all the possible two-electron processes, which result in a reaction in a single molecule. Here we neglect the back energy transfer from the RC mode to the HF mode. This means that HF mode is only excited by tunneling electrons, whereas the RC mode is excited either by tunneling electrons or by intermode anharmonic interaction with the HF mode in the excited states.

We decompose all the possible reaction channels into three classes A, B, and C.

$$
\begin{gathered}
\text { Process A: } \\
{[\nu: 0 \rightarrow 1](\nu: 1 \rightarrow 0 \mid \mathrm{RC}: 0 \rightarrow m) \times[\nu: 0 \rightarrow 1]} \\
\times(\nu: 1 \rightarrow 0 \mid \mathrm{RC}: m \rightarrow n)+[\nu: 0 \rightarrow 1][\nu: 1 \rightarrow 2] \\
\times(\nu: 2 \rightarrow 1 \mid \mathrm{RC}: 0 \rightarrow m)(\nu: 1 \rightarrow 0 \mid \mathrm{RC}: m \rightarrow n) \\
+[\nu: 0 \rightarrow 1][\nu: 1 \rightarrow 2] \times(\nu: 2 \rightarrow 0 \mid \mathrm{RC}: 0 \rightarrow n) .
\end{gathered}
$$

Here $[\nu: 0 \rightarrow 1]$ stands for a process where a tunnel electron excites the HF mode $\nu$ from the ground state $\nu=0$ to the first excited state $\nu=1 .(\nu: 1 \rightarrow 0 \mid \mathrm{RC}: 0 \rightarrow m)$ denotes a process where the HF mode decays from its first vibrational excited state $\nu=1$ to the ground state $\nu=0$ while the RC mode is excited from the ground state to any level within a bundle of levels which we collectively denote by $m$, as depicted in Fig. 3. This transfer of energy from the HF mode to the bundle $m$ is possible because of the anharmonic coupling between the

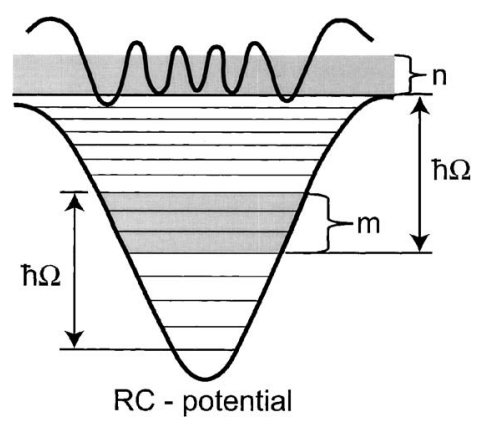

FIG. 3. Schematic illustration of bundle of intermediate excited and final states (gray areas) of the RC mode. See text for details.

two coordinates. Since both the RC mode and the HF mode have discrete energy levels ( $\hbar \omega_{m}$ and $n \hbar \Omega$, respectively) rather than a continuum of levels, energy conservation requires depositing some energy $\epsilon$ in the continuum of EHP excitations (for metals) or bulk phonon excitations. Thus, in general $\hbar \Omega=\hbar \omega_{m}+\epsilon$. The bundle of energy levels $m$ is defined so that excitation from any levels within the bundle can give rise to a reaction. This requires that the separation between the lowest level within the bundle $m$ and the top of the RC barrier must be equal to or smaller than $\hbar \Omega$. Furthermore, the separation between the highest level in the bundle $m$ and the lowest level in the reaction coordinate well must be at most $\hbar \Omega$ since this is the maximum possible energy transfer from the HF mode.

The first term in Eq. (12) describes a process where the HF mode is first excited by a tunneling electron, followed by a deexcitation accompanied with the transfer of energy (via anharmonic coupling) to the $\mathrm{RC}$ mode. This process is then repeated while the RC mode is excited to a level above the top of the barrier $E_{B}$. The second term describes an event where the HF mode is first excited to its second excited state $\nu=2$ by two inelastic-tunneling events, followed by two deexcitation processes where energy is transferred to the RC mode, which is again excited to a level above the top of the barrier $E_{B}$. The last term is allowed when the HF mode is anharmonic. In this case the second excited state of the HF mode can return to its ground state in a single step, while energy is transferred to the RC mode to overcome the barrier $E_{B}$. This is a two-electron version of a single-electron process shown in Fig. 2, in a sense that the RC mode undergoes the direct transition to the bundle $n$ without populating the intermediate bundle $m$.

$$
\begin{gathered}
\text { Process B: } \\
{[\nu: 0 \rightarrow 1](\nu: 1 \rightarrow 0 \mid \mathrm{RC}: 0 \rightarrow m)[\mathrm{RC}: m \rightarrow n]} \\
+[\mathrm{RC}: 0 \rightarrow m][\nu: 0 \rightarrow 1](\nu: 1 \rightarrow 0 \mid \mathrm{RC}: m \rightarrow n) .
\end{gathered}
$$

This is a process where both the HF mode and the RC mode are excited by tunneling electrons, and where transfer of energy from the HF mode to the RC mode again leads to the excitation of the latter above the barrier $E_{B}$. In this second process the top of the bundle $m$ is determined by the applied bias voltage, not by $\hbar \Omega$.

The processes A can induce molecular motion (reaction) if the potential barrier $E_{B}$ is lower than $2 \hbar \Omega$. This is the case 
for $\mathrm{NH}_{3}$ desorption from $\mathrm{Cu}(100)$ surface, where $\hbar \Omega=408 \mathrm{meV}\left(\mathrm{N}-\mathrm{H}\right.$ vibration) and $E_{B}=600 \mathrm{meV}^{22}$ On the other hand, processes B can occur if $\hbar \Omega+\hbar \omega_{m}>E_{B}$ (one electron excites the RC mode from the ground state to the $m$ state), or if $\hbar \Omega+\hbar \omega_{n}-\hbar \omega_{m}>E_{B}$ (the intermediate excited $m$ state, which results from the deexcitation of the $\nu$ mode, is further excited above $E_{B}$ by another tunneling electron). For instance, in the case of $\mathrm{NH}_{3}$ desorption from $\mathrm{Cu}(100)$, the overtone excitation of the umbrella mode (with the energy $\left.\hbar \omega_{U} \simeq 140 \mathrm{meV}\right)$ satisfies $\hbar \Omega_{\mathrm{N}-\mathrm{H}}+2 \hbar \omega_{U}>E_{B}$. Furthermore, the inelastic-tunneling fraction $\eta_{U} \simeq 4 \times 10^{-3}$ of the fundamental excitation of the umbrella mode is larger than $\eta_{\mathrm{N}-\mathrm{H}} \simeq 8 \times 10^{-4}$ for the $\mathrm{N}-\mathrm{H}$ mode, and the EHP damping rates $1 / \tau_{\mathrm{N}-\mathrm{H}}=0.26 \times 10^{12} / \mathrm{s}$ and $1 / \tau_{U}=0.04 \times 10^{12} / \mathrm{s}$. These calculated quantities using density-functional theory ${ }^{7}$ may not be accurate, but suggest that process $\mathrm{B}$ is a possible reaction pathway for $\mathrm{NH}_{3}$ desorption from $\mathrm{Cu}(100)$.

$$
\text { Process C: } \quad[\mathrm{RC}: 0 \rightarrow m][\mathrm{RC}: m \rightarrow n]
$$

is a reaction induced by a step-by-step direct excitation of the RC mode by two tunneling electrons, where each step involves multiple-vibrational excitations from $0 \rightarrow m$ and from $m \rightarrow n$. Thus, in contrast to processes $\mathrm{A}$ and $\mathrm{B}, \mathrm{C}$ does not involve the HF mode.

\section{REACTION RATE}

Using Eq. (1) the reaction rate due to all the two-electron processes described above is given by

$$
R_{\mathrm{RC}}=\frac{N_{1, m}}{\tau_{\nu(1 \rightarrow 0) \mathrm{RC}(m \rightarrow n)}}+\frac{N_{2,0}}{\tau_{(2 \rightarrow 0) \mathrm{RC}(0 \rightarrow n)}}+p_{\mathrm{RC}}^{m \rightarrow n} N_{0, m},
$$

where $m$ and $n$ stand for the bundle of vibrational levels in the RC-potential well (see Fig. 3). That is, when the RC mode is in any of the energy levels in the bundle $m$, the transfer of energy $\hbar \Omega$ from the HF mode to the RC-mode or direct excitation of the $\mathrm{RC}$ mode by inelastic tunneling is used to excite the RC mode to a level in the bundle $n$ above the barrier $E_{B}$.

The first term in Eq. (15) represents a subclass of the processes $\mathrm{A}$ and $\mathrm{B}$, and

$$
\frac{1}{\tau_{\nu(1 \rightarrow 0) \mathrm{RC}(m \rightarrow n)}}
$$

is the transition rate for the deexcitation of the $\mathrm{HF}$ mode $\nu=1$ while simultaneously exciting the RC mode from the bundle $m$ to the bundle $n$. The second term represents one process from class $\mathrm{A}$, and

$$
\frac{1}{\tau_{\nu(2 \rightarrow 0) \mathrm{RC}(0 \rightarrow n)}}
$$

is the overtone $(2 \rightarrow 0)$ deexcitation rate of the HF mode that enables the RC mode to be directly excited to the bundle $n$. The third term is due to the direct excitation of the RC mode to the bundle $n$ above $E_{B}$ by coherent multiple-vibrational excitation from its intermediate bundle $m$ that is populated either through decay of the HF mode (process B) or by the initial excitation of the RC mode from the ground state by a tunneling electron (process $\mathrm{C}$ ).

The rate equation for $N_{1, m}$ describing the $\mathrm{HF}$ mode in the first excited state and the RC mode in the intermediate bundle $m$ is given by

$$
\begin{aligned}
\frac{d N_{1, m}}{d t}= & p_{\nu} N_{0, m}+\frac{N_{2,0}}{\tau_{\nu(2 \rightarrow 1) \mathrm{RC}(0 \rightarrow m)}}+p_{\mathrm{RC}}^{0 \rightarrow m} N_{1,0}-2 p_{\nu} N_{1, m} \\
& -\frac{1}{\tau_{\nu}} N_{1, m}-\frac{1}{\tau_{\mathrm{RC}}} N_{1, m}-\frac{N_{1, m}}{\tau_{\nu(1 \rightarrow 0) \mathrm{RC}(m \rightarrow n)}}
\end{aligned}
$$

There are three processes which positively contribute to $N_{1, m}$ : from $N_{0, m}$ by a tunneling current $p_{\nu}$ to excite the HF mode, from $N_{2,0}$ via the energy transfer $1 / \tau_{\nu(2 \rightarrow 1) \mathrm{RC}(0 \rightarrow m)}$ to the RC mode through the deexcitation of the $\nu$ mode to the first excited state, and from $N_{1,0}$ by a tunneling electron $p_{\mathrm{RC}}^{0 \rightarrow m}=\eta_{\mathrm{RC}}^{0 \rightarrow m} I$, where $\eta_{\mathrm{RC}}^{0 \rightarrow m}$ represents the inelastic-tunneling fraction that excites the RC mode from the ground state to the intermediate excited state $m$ by a coherent multiplevibration excitation. The explicit form of $p_{\mathrm{RC}}^{0 \rightarrow m}$ (and $\eta_{\mathrm{RC}}^{0 \rightarrow m}$ ) could be obtained, for example, using a theory developed by Salam et al. ${ }^{13}$ Starting with the result for the probability that an electron tunnels inelastically via an adsorbate resonance by exciting the RC mode from the ground state to the $m$ th excited states, ${ }^{28}$ they derived an explicit expression for the rate of coherent multiple-vibrational excitation by inelasticelectron tunneling. It is noted here that Persson ${ }^{29}$ has predicted that when $\hbar \Omega / \Delta \ll 1$ and $\delta \epsilon / \Delta \ll 1$ (where $\Delta$ and $\delta \epsilon$ are the width of the relevant adsorbate-induced resonance state and the electron-vibration coupling, respectively), inelastic tunneling via multiple-vibrational excitation is negligible; a large width $\Delta$ implies a short electron-vibration interaction time resulting in a small probability for multivibrational excitation. This is indeed the case for $\mathrm{CO}$ chemisorbed on metal surfaces where $\Delta$ is about $1 \mathrm{eV}$ or more. On the other hand, for $\delta \epsilon / \Delta \approx 1$, multiple-vibrational excitation can be effective. It is also obvious that inelastic tunneling via multiple-vibrational excitation by a single electron is possible only if $\mathrm{eV}>n \hbar \omega$, where $n \geqslant 2$.

$\tau_{\mathrm{RC}}$ in Eq. (18) is the average time it takes for the RC mode to make a transition from the bundle $m$ to any lowerlying levels in the reaction coordinate well. This relaxation process may involve several transitions between vibrational levels. Since the spacing between vibrational levels of the RC mode may be very small, the vibrational transition $m \rightarrow m-1$ must involve the energy transfer to EHP or substrate phonons. Note also that $1 / \tau_{\nu(2 \rightarrow 1) \mathrm{RC}(0 \rightarrow m)}$ or $1 / \tau_{\nu(1 \rightarrow 0) \mathrm{RC}(0 \rightarrow m)}$ must involve the continuum of low-energy excitations (EHP or bulk phonons) in order to satisfy the energy conservation. However, the second excitation $m \rightarrow n$ of the RC mode above the barrier does not necessarily require additional low-energy excitations because of the continuum scattering states above $E_{B}$ associated with molecular motions. The damping rate $1 / \tau_{\mathrm{RC}}$ of the $\mathrm{RC}$ mode must be smaller than or comparable to $1 / \tau_{\nu}$ and/or tunneling current rate, since the RC mode should remain in the bundle $m$ to accept a sufficient energy either from the HF mode or from a tunneling electron. 
In a similar fashion we obtain

$$
\begin{aligned}
\frac{d N_{0, m}}{d t}= & \frac{N_{1,0}}{\tau_{\nu(1 \rightarrow 0) \mathrm{RC}(0 \rightarrow m)}}+\frac{1}{\tau_{\nu}} N_{1, m}+p_{\mathrm{RC}}^{0 \rightarrow m} N_{0,0}-\frac{1}{\tau_{\mathrm{RC}}} N_{0, m} \\
& -p_{\mathrm{RC}}^{m \rightarrow n} N_{0, m}-p_{\nu} N_{0, m}
\end{aligned}
$$

where $N_{0, m}$ is populated from $N_{1,0}$ at a rate $1 / \tau_{\nu(1 \rightarrow 0) \mathrm{RC}(0 \rightarrow m)}$ that represents the excitation of the RC mode to the $m$ states by the deexcitation of the HF mode to the ground state, and by a direct excitation of the RC mode from the initial state $N_{0,0}$. We also have

$$
\begin{aligned}
\frac{d N_{2,0}}{d t}= & 2 p_{\nu} N_{1,0}-\frac{2}{\tau_{\nu}} N_{2,0}-\frac{N_{2,0}}{\tau_{\nu(2 \rightarrow 1) \mathrm{RC}(0 \rightarrow m)}} \\
& -\frac{N_{2,0}}{\tau_{\nu(2 \rightarrow 0) \mathrm{RC}(0 \rightarrow n)}},
\end{aligned}
$$

where we used that the damping rate $1 / \tau_{\nu(2 \rightarrow 1)}$ of the $\nu=2$ state is twice $1 / \tau_{\nu}=1 / \tau_{\nu(1 \rightarrow 0)}$ of the $\nu=1$ state, ${ }^{30}$ and

$$
\begin{aligned}
\frac{d N_{1,0}}{d t}= & p_{\nu} N_{0,0}+\frac{2}{\tau_{\nu}} N_{2,0}+\frac{1}{\tau_{\mathrm{RC}}} N_{1, m}-2 p_{\nu} N_{1,0}-\frac{1}{\tau_{\nu}} N_{1,0} \\
& -p_{\mathrm{RC}}^{0 \rightarrow m} N_{1,0}-\frac{N_{1,0}}{\tau_{\nu(1 \rightarrow 0) \mathrm{RC}(0 \rightarrow \mathrm{m})}} .
\end{aligned}
$$

These coupled rate equations for $N_{1, m}, N_{0, m}, N_{2,0}$, and $N_{1,0}$ are solved using the basic assumption of a weak inelasticity, i.e., we neglect the electron-tunneling-induced depopulation in Eqs. (19) and (21). This assumption implies that we can neglect $-2 p_{\nu} N_{1, m}$ and $-2 p_{\nu} N_{1,0}$ in the right-hand side of Eqs. (18) and (21), respectively. For small adsorbed molecules on metal surfaces typically $\eta_{\nu}=1 \times 10^{-3}$ (see Refs. 24, 31, and 32) and $\tau_{\nu}=1 \times 10^{-12} \mathrm{~s}$ (see Ref. 25) and with the tunneling current of $1 \mathrm{nA}$, corresponding to $0.6 \times 10^{10} \mathrm{e} / \mathrm{s}$, one obtains $p_{\nu} \tau_{\nu}=6 \times 10^{-6} \ll 1$. On the other hand, large molecules, or molecules separated from the (metal) substrate by a thin nanometer thick spacer layer, or molecules adsorbed on semiconductors, may have vibrational lifetimes of order of nanoseconds or longer. In such cases, if we assume the tunneling current of $100 \mathrm{nA}$ and $\eta_{\nu}=10^{-2}$, one obtains $p_{\nu} \tau_{\nu}>1$. In this case we must include more terms (higher multielectron processes) in the rate equations studied above, and the HF mode can no longer be treated as a three level system $(\nu=0,1,2)$, but higher levels should be included. Furthermore, in addition to inelastic-tunneling events where the tunneling electrons excite the adsorbate to higher levels, one must include inelastic-tunneling processes where tunneling electrons absorb vibrational quanta from the excited molecules.

The solutions of Eqs. (18)-(21) give the reaction rate for the process A;

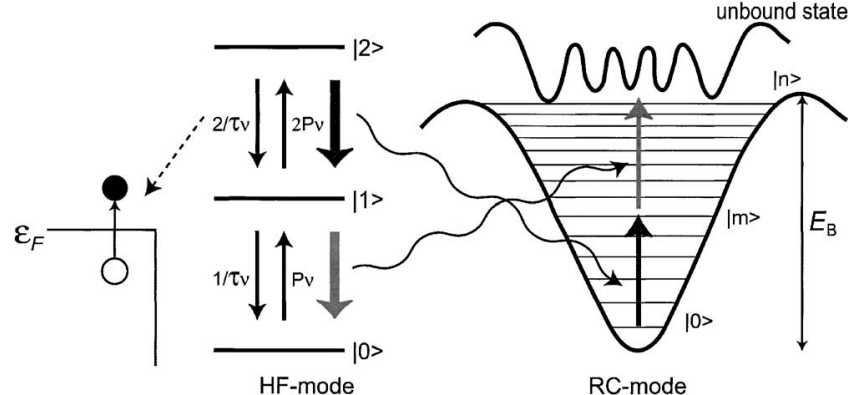

FIG. 4. Twice decays to the first excited state (black downward arrow) or the step-by-step decay from the second excited state of the HF mode to the ground state (blue and green downward arrows) through intermode energy transfer to the RC mode, in competing with electron-hole pair (EHP) excitations.

$$
\begin{aligned}
R_{\mathrm{RC}}^{\mathrm{A}}= & {\left[\frac{\tau_{1,0}}{\tau_{\nu(1 \rightarrow 0) \mathrm{RC}(0 \rightarrow m)}} \frac{\tau_{1, m}}{\tau_{\nu(1 \rightarrow 0) \mathrm{RC}(m \rightarrow n)}} \tau_{\mathrm{RC}}\right.} \\
& +2 \frac{\tau_{2,0}}{\tau_{\nu(2 \rightarrow 1) \mathrm{RC}(0 \rightarrow m)}} \frac{\tau_{1, m}}{\tau_{\nu(1 \rightarrow 0) \mathrm{RC}(m \rightarrow n)}} \tau_{1,0} \\
& \left.+2 \frac{\tau_{2,0}}{\tau_{\nu(2 \rightarrow 0) \mathrm{RC}(0 \rightarrow n)}} \tau_{1,0}\right] p_{\nu}^{2},
\end{aligned}
$$

where

$$
\begin{aligned}
& \frac{1}{\tau_{1,0}}=\frac{1}{\tau_{\nu}}+\frac{1}{\tau_{\nu(1 \rightarrow 0) \mathrm{RC}(0 \rightarrow m)}}, \\
& \frac{1}{\tau_{1, m}}=\frac{1}{\tau_{\nu}}+\frac{1}{\tau_{\mathrm{RC}}}+\frac{1}{\tau_{\nu(1 \rightarrow 0) \mathrm{RC}(m \rightarrow n)}}, \\
& \frac{1}{\tau_{2,0}}=\frac{2}{\tau_{\nu}}+\frac{1}{\tau_{\nu(2 \rightarrow 1) \mathrm{RC}(0 \rightarrow m)}}+\frac{1}{\tau_{\nu(2 \rightarrow 0) \mathrm{RC}(0 \rightarrow n)}}
\end{aligned}
$$

represent the total decay rate of $N_{1,0}, N_{1, m}$, and $N_{2,0}$, respectively. The first term in this equation corresponds to an activation of the RC mode via the step-by-step decay of the $\nu=2$ mode, while the second term represents a twice deexcitation of the $\nu=1$ mode. These two reaction paths are schematically illustrated in Fig. 4. The last term represents the process shown in Fig. 5. Here the lifetime of the RC mode plays no role since the RC mode is directly excited to the

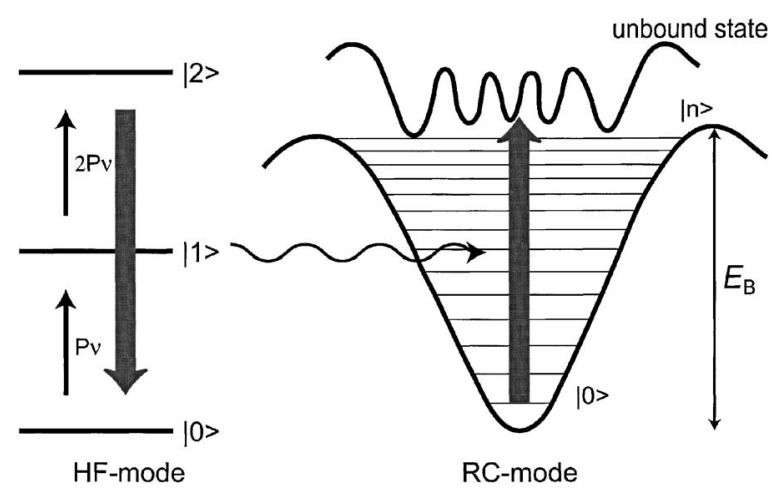

FIG. 5. In the presence of the anharmonicity of the HF mode, the direct deexcitation to the ground state enables the RC mode to be excited above $E_{B}$ without populating its intermediate states. 


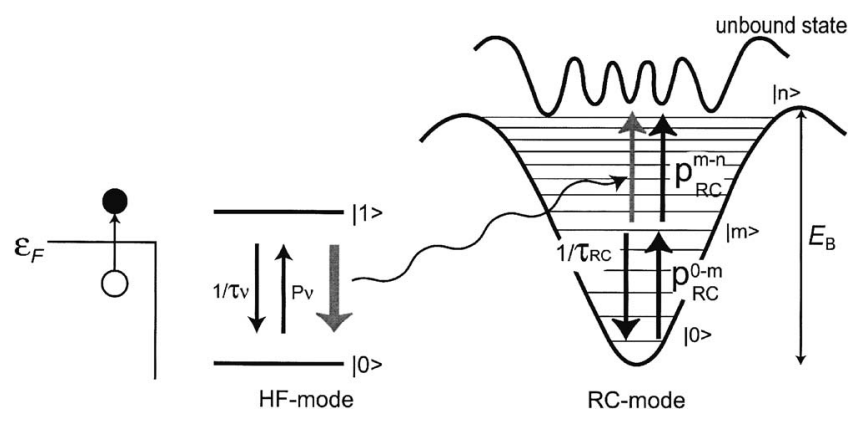

FIG. 6. Cooperative and direct excitation processes. (a) Each tunneling electron excites the $\nu$ and the RC modes to the first excited state and the intermediate excited state $m$, indicated by $p_{\nu}$ and $p_{\mathrm{RC}(0 \rightarrow m)}$ (black upward arrows), respectively. Decay of the $\nu$ mode further excites the RC mode from $m$ to the unbound states $n$ above the barrier (green arrow). (b) Decay of the $\nu$ mode excites the RC mode from the ground state to the $m$ th excited state, and another electron excites the RC mode from $m$ to the unbound states above the barrier (not shown in this figure). (c) The RC mode excited to $m$ by $p_{\mathrm{RC}(0 \rightarrow m)}$ is activated above $E_{B}$ by another tunneling electron (black upward arrow).

bundle $n$ above $E_{B}$ by the deexcitation of the HF mode from $\nu=2$ to $\nu=0$. If we assume, as is typically the case, that the population damping rates $1 / \tau_{\nu}$ and $1 / \tau_{\mathrm{RC}}$ are much faster than any intermode coupling rates $1 / \tau_{\nu, \mathrm{RC}}$, to leading order in $\tau_{\nu, \mathrm{RC}} / \tau_{\nu}$ and $\tau_{\nu, \mathrm{RC}} / \tau_{\mathrm{RC}}$, Eq. (22) is simplified to

$$
\begin{aligned}
R_{\mathrm{RC}}^{\mathrm{A}}= & \left(\frac{1}{\tau_{\nu}}+\frac{1}{\tau_{\mathrm{RC}}}\right)^{-1} \\
& \times\left(\frac{\tau_{\mathrm{RC}}}{\tau_{\nu}} \frac{1}{\tau_{\nu(1 \rightarrow 0) \mathrm{RC}(0 \rightarrow m)}} \frac{1}{\tau_{\nu(1 \rightarrow 0) \mathrm{RC}(m \rightarrow n)}}\right)\left(\eta_{\nu} \tau_{\nu}\right)^{2} I^{2} \\
& +\frac{1}{\tau_{\nu(2 \rightarrow 1) \mathrm{RC}(0 \rightarrow m)}} \frac{1}{\tau_{\nu(1 \rightarrow 0) \mathrm{RC}(m \rightarrow n)}}\left(\eta_{\nu} \tau_{\nu}\right)^{2} I^{2} .
\end{aligned}
$$

The reaction rate due to the cooperative process $\mathrm{B}$ depicted in Fig. 6 is obtained as

$$
\begin{gathered}
R_{\mathrm{RC}}^{\mathrm{B}}=\left(\tau_{1,0}+\tau_{\mathrm{RC}}\right) \frac{\tau_{1, m}}{\tau_{\nu(1 \rightarrow 0) \mathrm{RC}(m \rightarrow n)}} p_{\nu} p_{\mathrm{RC}}^{0 \rightarrow m} \\
+\frac{\tau_{1,0}}{\tau_{\nu(1 \rightarrow 0) \mathrm{RC}(0 \rightarrow m)}} \tau_{\mathrm{RC}} p_{\nu} p_{\mathrm{RC}}^{m \rightarrow n} \\
\simeq\left(\frac{1}{\tau_{\nu(1 \rightarrow 0) \mathrm{RC}(m \rightarrow n)}} \eta_{\mathrm{RC}}^{0 \rightarrow m}\right. \\
\left.+\frac{1}{\tau_{\nu(1 \rightarrow 0) \mathrm{RC}(0 \rightarrow m)}} \eta_{\mathrm{RC}}^{m \rightarrow n}\right) \tau_{\nu} \eta_{\nu} \tau_{\mathrm{RC}} I^{2}
\end{gathered}
$$

where the first term represents a process in which two tunneling electrons excites the $\mathrm{HF}$ and $\mathrm{RC}$ mode to the first excited and the $m$ th intermediate excited state, respectively, indicated by $p_{\nu}$ and $p_{\mathrm{RC}}^{0 \rightarrow m}$ (black upward arrows in Fig. 6), respectively, and the decay of the $\mathrm{HF}$ mode to the ground state further excites the RC mode from $m$ to the unbound states $n$ above the barrier (green arrow). This reaction path requires a longer lifetime of the $\mathrm{RC}$ mode than that of the $\mathrm{HF}$ mode, otherwise the excited RC mode leaves the bundle $m$ before obtaining the energy from the HF mode necessary in order to excite it above the barrier $E_{B}$. Another tunneling electron then activates the RC mode above the barrier before the intermediate state of the RC mode is deexcited below the critical level (below which a tunneling electron is unable to excite the mode above the barrier). The second term involves the reverse order of the excitation processes, in which energy transfer from the HF mode (excited by a tunneling electron) to the RC mode excites the latter from the ground state to the intermediate bundle $m$, followed by the excitation of the RC mode to a state above the barrier $E_{B}$ by another tunneling electron. In this case too, the RC mode must be so long lived that it with large enough probability remains in the excited state before the next excitation (by another tunneling electron) occurs.

The reaction rate of the process $\mathrm{C}$ (also shown in Fig. 6), which does not require any help of the HF mode, is simply given by

$$
R_{\mathrm{RC}}^{\mathrm{C}}=\tau_{\mathrm{RC}} \eta_{\mathrm{RC}}^{0 \rightarrow m} \eta_{\mathrm{RC}}^{m \rightarrow n} I^{2} .
$$

Because of the incoherent two-step excitation, the reaction rate is proportional to $\tau_{\mathrm{RC}}$. Equation (29) can be extended to an incoherent multiple single-step ladder climbing in the truncated oscillator model by replacing $p_{\mathrm{RC}(0 \rightarrow m)}$ with $p_{\mathrm{RC}(0 \rightarrow 1)}^{m}=p_{\mathrm{RC}}^{m}$, and $p_{\mathrm{RC}(m \rightarrow n)}$ with $p_{\mathrm{RC}}^{n-m}$, where $p_{\mathrm{RC}}=\eta_{\mathrm{RC}} I$. This gives $R_{\mathrm{RC}}^{\mathrm{C}}=\tau_{\mathrm{RC}}^{n-1} \eta_{\mathrm{RC}}^{n} I^{n}$, where $\tau_{\mathrm{RC}}$ is the lifetime of a single-vibrational level, not as the bundle of vibrational states, and $n$ is a number of the vibrational levels in the potential well, in agreement with Gao et al. ${ }^{8}$

Now, let us examine which two-electron process becomes dominant over others depending on the inelastictunneling current $\left(p_{\nu}, p_{\mathrm{RC}}\right)$, the vibrational damping $\left(\tau_{\nu}, \tau_{\mathrm{RC}}\right)$, and the intermode coupling $\left(\tau_{\nu, \mathrm{RC}}\right)$. For $p_{\nu} \tau_{\nu} \gg p_{\mathrm{RC}} \tau_{\mathrm{RC}}$, the processes A [Eq. (26)] becomes dominant over the processes B [Eq. (28)] and C [Eq. (29)]. Among the three different reaction pathways in the processes $A$ the population of the second excited state of the HF mode is extremely small for $1 / \tau_{\nu} \gg 1 / \tau_{\mathrm{RC}}$ so that the excitation of the RC mode via the step-by-step decay (the second; $\nu=2 \rightarrow 1 \rightarrow 0$ ) is unlikely compared to the twice decay of the first excited state [the first term; $2 \times(\nu=1 \rightarrow 0)]$. In the opposite limit where the HF mode remains long enough in the first excited state, there is higher probability for it to be excited to the $\nu=2$ state so that the step-by-step decay process of the HF mode [the first term of Eq. (12)] becomes dominant in the reaction rate. Thus, the ratio of the vibrational damping rate of the $\nu$ mode and the RC mode determines the reaction pathway. Decay in one single step of a doubly excited HF mode [the last term of Eq. (26)] seems unlikely due to the inefficiency of the anharmonicity of the HF and the RC modes. This is consistent with density-functional theory (DFT) calculation for the desorption of $\mathrm{NH}_{3}$ from $\mathrm{Cu}(100){ }^{33}$

According to the experimental results of $\mathrm{NH}_{3}$ motion (translation and desorption) on $\mathrm{Cu}(100)$ (Ref. 22) the total reaction probability exhibits a threshold at $\sim 400 \mathrm{mV}$ corresponding to excitation of the $\mathrm{N}-\mathrm{H}$ mode at low tunneling current $I$ below $0.5 \mathrm{nA}$, where translation is induced by a single-electron process. An additional threshold appears at 
$\sim 270 \mathrm{mV}$, consistent with the energy of two umbrella mode quanta. This suggests that process B [Eq. (28)] (excitation of both the $\mathrm{N}-\mathrm{H}$ mode and an overtone of the umbrella mode by tunneling electrons) contributes to the two-electron process of $\mathrm{NH}_{3}$ desorption. The five times larger inelastictunneling probability and longer lifetime of the umbrella mode than those of the $\mathrm{N}-\mathrm{H}$ mode calculated by Lorente et $a l^{7}$ also support the involvement of the process B. This implies that the dominant reaction path of $\mathrm{NH}_{3}$ desorption changes from the process A to B with an increase in tunneling current.

As for the process $\mathrm{C}$ it has been predicted that the incoherent multiple excitation rate dominates actually over the coherent one when the vibrational relaxation rate is much larger than the tunneling rate through the resonance (see Ref. 13). Equation (29) includes both vibrational excitation mechanisms. The indirect excitation of the RC mode through intermode coupling with the HF mode will dominate over the stepwise ladder climbing of the RC mode if the lifetime of the RC mode is very short. This is certainly the case for hopping of $\mathrm{CO}$ on $\mathrm{Pd}(110),{ }^{17} \mathrm{NH}_{3}$ on $\mathrm{Cu}(100),{ }^{22}$ and for the rotation of $\mathrm{C}_{2} \mathrm{H}_{2}$ on $\mathrm{Cu}(100),{ }^{18}$ and for the desorption of $\mathrm{NH}_{3}$ from $\mathrm{Cu}(100){ }^{22}$

The reaction pathways to induce and to control the dynamical motion of a single molecule by two tunneling electron processes require more systematic experiments, including studies of the current and bias voltage dependences of the reaction yield, and theoretical efforts in order to gain a deeper insight into the coupling between the initial vibrational (or electronic) excitation, and the nuclear motion of adsorbates along the relevant reaction coordinates. ${ }^{34}$

\section{SUMMARY}

We have discussed how the excitation of high-frequency modes in adsorbed molecules may result in motion (e.g., rotation, translation, or dissociation) of the molecules. Our study is based on rate equations and considers one- and twoelectron processes. The parameters which enter in the rate equations are inelastic-tunneling rates, vibrational relaxation rates and, most importantly, intermode anharmonic coupling rates $1 / \tau_{\nu, \mathrm{RC}}$. Some of these rate coefficients can be obtained experimentally, e.g., from studies of vibrational relaxation using pump and probe methods or other techniques. The intramolecular transfer rates are harder to obtain from independent experiments, but may be estimated from potentialenergy surfaces calculated using the density-functional method. Conversely, from studies of the type reported in this paper it should be possible to obtain intramolecular transition rates such as $1 / \tau_{\nu(1 \rightarrow 0) \mathrm{RC}(0 \rightarrow m)}$ directly from the experimental data, and gain some understanding on how these important quantities depend on the modes involved and the substrate. For adsorbate vibrational relaxation, involving the transfer of the energy to the substrate as low-energy electronic excitations or bulk phonons, such an understanding already exists based on theoretical calculations and on experimental results obtained using infrared spectroscopy, inelastic helium atom scattering, and various pump and probe spectroscopies. The information described above will clarify the microscopic mechanism of how to selectively manipulate single adsorbed molecules at surfaces.

\section{ACKNOWLEDGMENTS}

We would like to thank M. Persson for critical reading of the manuscript and comments. We are also very grateful to W. Ho, M. Kawai, and J. I. Pascual for private communications. This work was supported by Japan Society for the Promotion of Science (JSPS); Grant-in-Aid for Basic Research (B) No. 15310071 (H.U.), Grant-in-Aid-for Young Scientists (B) No. 16740173 (T.M.), Postdoctoral Fellowship for North American and European Researchers, No. PE04024 (N.L.), and Invitation Fellowship Program for Research in Japan, No. S-04034 (B.P.).

${ }^{1}$ B. C. Stipe, M. A. Rezaei, and W. Ho, Science 280, 1732 (1998)

${ }^{2}$ W. Ho, J. Chem. Phys. 117, 11033 (2002).

${ }^{3}$ S.-W. Hla and K.-H. Rieder, Annu. Rev. Phys. Chem. 54, 307 (2003).

${ }_{5}^{4}$ H. Ueba, Surf. Rev. Lett. 10, 771 (2003).

${ }^{5}$ H. Ueba and B. N. J. Persson, Surf. Sci. 566-568, 1 (2004).

${ }^{6}$ H. Ueba, Appl. Surf. Sci. 273, 565 (2004).

${ }^{7}$ N. Lorente, R. Rurali, and H. Tang, J. Phys.: Condens. Matter 17, S1049 (2005).

${ }^{8}$ S. Gao, M. Persson, and B. I. Lundqvist, Solid State Commun. 84, 271 (1992); Phys. Rev. B 55, 4825 (1997).

${ }^{9}$ R. E. Walkup, D. M. Newns, and Ph. Avouris, J. Electron Spectrosc. Relat. Phenom. 64/65, 523 (1993); Phys. Rev. B 48, 1858 (1993).

${ }^{10}$ S. G. Tikhodeev and H. Ueba, Phys. Rev. B 70, 125414 (2004).

${ }^{11}$ D. M. Eigler, C. P. Lutz, and W. E. Rudge, Nature (London) 352, 600 (1991).

${ }^{12}$ B. N. J. Persson and Ph. Avouris, Surf. Sci. 390, 45 (1997).

${ }^{13}$ G. P. Salam, M. Persson, and R. E. Palmer, Phys. Rev. B 49, 10655 (1994).

${ }^{14}$ B. C. Stipe, M. A. Rezaei, W. Ho, S. Gao, M. Persson, and B. I. Lundqvist, Phys. Rev. Lett. 78, 4410 (1997).

${ }^{15}$ Y. Kim, T. Komeda, and M. Kawai, Phys. Rev. Lett. 89, 126104 (2002).

${ }^{16}$ T. Komeda, Y. Kim, M. Kawai, B. N. J. Persson, and H. Ueba, Science 295, 2055 (2002)

${ }^{17}$ B. C. Stipe, M. A. Rezaei, and W. Ho, Phys. Rev. Lett. 81, 1263 (1998).

${ }^{18}$ B. N. J. Persson and H. Ueba, Surf. Sci. 502-503, 18 (2002).

${ }^{19}$ B. C. Stipe, M. A. Rezaei, and W. Ho, Science 279, 1907 (1998).

${ }^{20} \mathrm{~W}$. Ho (private communication).

${ }^{21}$ D. Teillet-Billy, J. P. Gauyacq, and M. Persson, Phys. Rev. B 62, R13306 (2000).

${ }^{22}$ J. I. Pascual, N. Lorente, Z. Song, H. Conrad, and H.-P. Rust, Nature (London) 423, 525 (2003).

${ }^{23}$ Y. Kim, C. Matsumoto, T. Okawa, Y. Saino, and M. Kawai (private communication)

${ }^{24}$ B. N. J. Persson and A. Baratoff, Phys. Rev. Lett. 59, 339 (1987).

${ }^{25}$ B. N. J. Persson and M. Persson, Solid State Commun. 36, 175 (1980).

${ }^{26}$ M. Head-Gordon and J. Tully, J. Chem. Phys. 96, 3939 (1992).

${ }^{27}$ H.-C. Chang and G. E. Ewing, Phys. Rev. Lett. 65, 2125 (1990).

${ }^{28}$ J. W. Gadzuk, Phys. Rev. B 44, 13466 (1991).

${ }^{29}$ B. N. J. Persson, Phys. Scr. 38, 282 (1987).

${ }^{30}$ P. Jakob and B. N. J. Persson, J. Chem. Phys. 109, 8641 (1998).

${ }^{31} \eta_{\nu}$ of the $\mathrm{C}-\mathrm{O}$ stretch mode of $\mathrm{CO}$ on $\mathrm{Cu}(111)$ is $5 \times 10^{-3}$ by M. Persson, Philos. Trans. R. Soc. London, Ser. A 362, 1173 (2004).

${ }^{32} \eta_{\nu}$ of the $\mathrm{N}-\mathrm{H}$ stretch mode of $\mathrm{NH}_{3}$ on $\mathrm{Cu}(100)$ is $8 \times 10^{-4}$ in Ref. 7

${ }^{33}$ N. Lorente and J. I. Pascual, Philos. Trans. R. Soc. London, Ser. A 362, 1227 (2004).

${ }^{34}$ In preparing the revised version of this manuscript we become aware about the dissociation and desorption of single chlorobenzene molecule on $\mathrm{Si}(111)-7 \times 7$ surface by P. A. Sloan and R. E. Palmer, Nature (London) 434, 367 (2005). In this paper a two-electron mechanism was proposed which couples vibrational excitation and dissociative electron attachment forming temporary negative-ion state. 